https://doi.org/10.1590/198053146526

\title{
LA DIMENSIÓN CURRICULAR COMO COMPONENTE DEL CONOCIMIENTO DIDÁCTICO DEL CONTENIDO'
}

Lina Melo'

Ana Caballero"

Luis Manuel Soto-Ardila"l

David Melo'v

\section{Resumen}

Se describe un estudio de caso centrado en el currículo de una profesora de física de bachillerato durante dos años consecutivos sobre la enseñanza del campo eléctrico, abordado desde tres niveles: declarativo, diseño y acción. La caracterización del conocimiento curricular se realiza bajo la perspectiva del conocimiento didáctico del contenido (CDC), utilizando como técnica el análisis del contenido. Los resultados muestran una alta tendencia tradicional en cada uno de los niveles considerados. Después de la intervención, el nivel de diseño es el que presenta los mayores cambios, notándose un incremento considerable en la tendencia intermedia. Estos resultados muestran la importancia del diseño curricular en los procesos de concreción del CDC.

PLAN DE ESTUDIOS • DIDÁCTICA • ENSEÑANZA • FÍSICA

\section{THE CURRICULAR DIMENSION AS A COMPONENT OF THE DIDACTIC KNOWLEDGE OF THE CONTENT}

\begin{abstract}
We describe a case study focused on the knowledge of the curriculum of a high school physics teacher, two consecutive years on electric field teaching. We used three levels to characterize the knowledge of the curriculum: declarative, design and action. The characterization of the knowledge of the curriculum was doing from pedagogical content knowledge (PCK), through the content analysis as a technique. The results show a high traditional tendency in each of the levels considered. After the intervention, the level of design represents the biggest changes, where intermediate trend increases. These results show the importance of curriculum design of education, in the process of realization of the PCK.
\end{abstract}

\section{CURRICULUM・DIDACTICS・TEACHING・PHISICS}

1 Esta investigación fue financiada por el Fondo Europeo de Desarrollo Regional y Junta de Extremadura. Proyecto GR18115, GR18004.L. Melo agradece al Proyecto EDU2016-77007-R (AEI / FEDER, UE) del Ministerio de Ciencia, Innovación y Universidades de España.

I Universidad de Extremadura, Departamento de Didáctica de las Ciencias Experimentales y las Matemáticas; Facultad de Educación, Badajoz, España; http://orcid.org/0000-0003-4771-058X; Ivme/o@unex.es

II Universidad de Extremadura, Departamento de Didáctica de las Ciencias Experimentales y las Matemáticas; Facultad de Educación, Badajoz, España; http://orcid.org/0000-0001-7538-3833; acabcar@unex.es

III Universidad de Extremadura, Departamento de Didáctica de las Ciencias Experimentales y las Matemáticas; Facultad de Educación, Badajoz, España; http://orcid.org/0000-0002-1041-4077; /uismanue/@unex.es

IV Universidad Pedagógica y Tecnológica de Colombia; Facultad de Educación, Colombia; http://orcid.org/0000-0003-3699-0993; david.me/o01@uptc.edu.co 


\section{LES PROGRAMMES SCOLAIRES EN TANT QUE COMPOSANTES DE LA CONNAISSANCE DIDACTIQUE DU CONTENU}

\section{Résumé}

Cet article décrit une étude de cas au long de deux années concernant le programme d'études sur l'enseignement du champ électrique d'une professeure de physique du secondaire, abordé selon trois niveaux: déclaratif, de la planification et de l'action. La caractérisation des connaissances des programmes se place dans la perspective de la connaissance didactique $d u$ contenu (CDC) s'appuyant sur la technique de l'analyse de contenu. Les résultats indiquent une forte tendance traditionnelle à tous les niveaux considérés. Après l'intervention, le niveau de la planification a été celui qui a présenté les plus grands changements, avec une augmentation considérable de la tendance intermédiaire. Ces résultats montrent l'importance de l'esquisse des programmes scolaires dans les processus de mise en ouvre du CDC.

PROGRAMMES • DIDACTIQUE • ENSEIGNEMENT • PHYSIQUE

\section{A DIMENSÃO CURRICULAR COMO COMPONENTE DO CONHECIMENTO DIDÁTICO DO CONTEÚDO}

\section{Resumo}

Descreve-se um estudo de caso centrado no currículo de uma professora de física do ensino médio durante dois anos consecutivos sobre o ensino do campo elétrico, abordado a partir de três níveis: declarativo, planejamento e ação. A caracterização do conhecimento curricular é realizada da perspectiva do conhecimento didático do conteúdo (CDC), utilizando como técnica a análise do conteúdo. Os resultados mostram uma alta tendência tradicional em cada um dos níveis considerados. Depois da intervenção, o nível de planejamento apresenta as maiores mudanças, notando-se um aumento considerável na tendência intermediária. Esses resultados mostram a importância do desenho curricular nos processos de concretização do CDC.

CURRÍCULO • DIDÁTICA • ENSINO • FÍSICA 
L TRABAJO QUE PRESENTAMOS SE ARTICULA BAJO LA PROPUESTA DEL CONOCIMIENTO Didáctico del Contenido (CDC) y forma parte de un estudio más amplio cuya intención es mostrar el desarrollo de este tipo de conocimiento con profesores de física de bachillerato en ejercicio. De acuerdo con Shulman (1986), el CDC es desarrollado por los profesores para ayudar a otros a aprender, y es construido mientras ellos enseñan tópicos específicos de su área de saber (ABELL, 2007). Las propuestas imperantes sobre este tipo de conocimiento han diferenciado dos posturas; aquellas en las cuales el CDC es considerado un conocimiento base más del conocimiento profesional del profesor, y otras donde es el resultado de la mediación dialógica (pasiva o activa) que aflora y se reconfigura en tanto se enseña y reflexiona, en y sobre lo que se enseña. Desde ambas perspectivas, el CDC se describe a través de: orientaciones y concepciones sobre la enseñanza de las ciencias; conocimiento curricular; conocimiento del aprendizaje y las ideas de los estudiantes; las estrategias de enseñanza; y la evaluación en ciencias (MAGNUSSON; KRAJCIK; BORKO, 1999).

Las nuevas exigencias y retos que confluyen en la enseñanza nos han llevado a explorar la relación, de lo sicopedagógico al conocimiento didáctico del contenido, lo cual ha dado paso a la inclusión del dominio afectivo dentro del CDC. Los estudios realizados en esta línea sugieren que los profesores, además de destacarse por lo cognitivo, por sus estrategias de enseñanza y su eficacia para lograr aprendizaje asertivo, también están llenos de actitudes y emociones positivas hacia sí mismos, hacia su trabajo y hacia sus alumnos (MELLADO et al., 2014), 
las cuales influyen en los procesos de enseñanza de los distintos contenidos en ciencias.

A pesar de estas nuevas exigencias, los estudios sobre el CDC se han centrado en caracterizar los procesos implicados en el desarrollo de este tipo de conocimiento. Mediante investigaciones empíricas se muestra cómo se conectan los diferentes aspectos de este conocimiento y cómo puede influir una componente en el crecimiento de las otras (HENZE; VAN DRIEL; VERLOOP, 2008; PARK; CHEN, 2012; VERDUGO-PERONA; SOLAZ-PORTOLÉS; SANJOSÉ-LÓPEZ, 2017).

Los principales mecanismos para propiciar el desarrollo han sido: reflexión orientada, tanto individual como en equipo; desarrollo de trabajos o propuestas de enseñanza; diseños curriculares y talleres de formación profesional. Hasta ahora los resultados nos sugieren que faltan herramientas que permitan dilucidar, en conjunto, las relaciones que describen el CDC, y sobre todo faltan representaciones holísticas de este conocimiento, independientes de los modelos que lo describen.

Convencidos del papel integral de CDC en el desarrollo profesional, diseñamos el estudio que describiremos a continuación, a través del cual caracterizamos el CDC de una profesora de física de Bachillerato desde lo que declara, diseña y hace sobre la enseñanza del campo eléctrico, durante dos años consecutivos después de un programa de intervención. Los resultados que presentamos corresponden a la categoría conocimiento curricular. También contribuimos a mostrar una forma de representar este tipo de conocimiento y sus cambios (SOLÍS; PORLÁN; RIVERO, 2012).

\section{EL PAPEL DEL CURRÍCULO EN EL CDC EN FÍSICA}

Los trabajos de Contreras (2008, 2009), Friedrischen et al. (2009, 2011), Schneider y Plasman (2011), Solís, Porlán y Rivero (2012) entre otros, nos han servido de referencia en esta categoría. Contreras $(2008 ; 2009)$ a través de un estudio con profesores chilenos de secundaria, por medio de una metodología cualitativa, describe las creencias curriculares y las creencias de actuación curricular frente a los contenidos, la metodología y la evaluación, además de establecer una relación aproximada entre el pensamiento y la actuación. Los resultados muestran una mezcla de tendencias frente a qué y cómo enseñar; una tendencia tradicional sobre con qué y cómo evaluar; y varias inconsistencias entre lo que consideran los profesores que deben hacer y lo que hacen realmente en el aula. El estudio indica que al mirar solo lo que los profesores piensan sobresale una tendencia constructivista, a diferencia de aquella que describe sólo la actuación.

Friedrichsen y Dana (2003), Friedrischen, Van Driel y Abell (2011) y Friedrischen et al. $(2009,2011)$ analizan la influencia de las orientaciones curriculares sobre la visión de la enseñanza de las ciencias. A partir del análisis del aula identificaron dos categorías: (a) las orientaciones centradas en el profesor (rigor didáctico y rigor académico) y (b) las orientaciones sobre la base de los esfuerzos de las reformas y proyectos curriculares asociados (proceso, actividades dirigidas, descubrimiento, cambio conceptual, investigación, proyectos). 
Cheung y Wong (2002) identificaron cinco orientaciones curriculares: procesos cognitivos, currículo como tecnología, humanista, currículo para la reconstrucción social y racionalismo académico. Diversos autores, entre ellos Stenhouse (1984) y Vázquez et al. (2012), han hecho una diferenciación entre la teoría técnica del currículum, la teoría práctica y la teoría crítica del currículum.

Schneider y Plasman (2011) señalan que los estudios específicos sobre el CDC en ciencias reportan falta de reflexión sobre las ideas científicas incluidas en sus instrucciones o los recursos disponibles para su enseñanza, lo que incrementa la dependencia de los materiales curriculares, como el libro de texto. Friedrichsen et al. (2009) indican que la revisión de los planes de estudio en grupos de profesores es un ejercicio fundamental para incrementar el conocimiento de los profesores sobre el currículo. En todos los casos las orientaciones curriculares no son el marco de análisis de las investigaciones, sino los fundamentos epistemológicos, psicológicos y sociales que soportan la didáctica como cuerpo de conocimiento.

Justi y van Driel (2005) y Orleans (2010) indican que los factores facilitadores del cambio del CDC se encuentran relacionados con aspectos de la componente conocimiento curricular en relación con el conocimiento sobre los estudiantes. Sin embargo, Henze, van Driel y Verloop (2008) muestran es sus estudios cómo el conocimiento curricular no muestra cambios a diferencia de todas las otras componentes del CDC. Concluyen que es un conocimiento que se relaciona de forma consistente con el conocimiento sobre las estrategias y los estudiantes.

Lee y Luft (2008) señalan la falta de estudios sobre el CDC con relación al conocimiento que los profesores tienen sobre los recursos. En nuestro estudio consideramos este aspecto como elemento del conocimiento curricular, aunque los autores lo posicionan como una componente más del CDC para resaltar su importancia y el olvido que sobre este aspecto han mostrado las investigaciones. Los resultados indican que el conocimiento que los profesores tienen sobre los recursos les permite hacer una instrucción relevante para sus estudiantes. Además, este conocimiento impacta sobre la organización curricular, la selección de estrategias de enseñanza y la utilización de las evaluaciones.

Los estudios de Seung (2013) con profesores, con y sin experiencia, muestran que los profesores con experiencia construyen los objetivos de sus asignaturas en función del conocimiento que tienen de los estudiantes, mientras que los profesores noveles lo construyeron en función de su conocimiento declarativo sobre el contenido. Por otro lado, el tipo de objetivos utilizados en los diversos contextos de enseñanza fue fundamental para propiciar comprensión sobre cómo enseñar el contenido. Los objetivos que menos se referencian son los debidos a la enseñanza, le siguen los referidos a los contenidos y en mayor medida los curriculares

Finalmente, Solís, Porlán y Rivera (2012) describen una forma particular de representar el conocimiento curricular del profesorado de ciencias y su evolución. Para cada categoría se construyeron "itinerarios de progresión” fundamentados en los modelos didácticos, los cuales sirvieron como referente para clasificar cada unidad de información extraída de los diversos instrumentos. Este proceso permitió construir una lectura visual e identificar la tendencia de cada profesor 
a través de las diferentes frecuencias asignadas a cada categoría. Los resultados muestran que los profesores, cuando se encuentran en la fase de formación teórica, expresan mayoritariamente referencias sobre el currículo centradas en el modelo didáctico de transición y el de referencia. En el momento de diseñar la práctica del aula, incrementan las unidades clasificadas en los modelos de transición y tradicional.

A pesar de la multiplicidad de definiciones y categorizaciones seguidas en las distintas investigaciones revisadas, los autores recomiendan centrar el análisis de las orientaciones sobre el currículo y en elementos más generales como: creencias sobre los propósitos de enseñanza de las ciencias, creencias acerca de la naturaleza de la ciencia y creencias sobre enseñanza y aprendizaje de las ciencias. La diversidad de categorías utilizadas para describir el conocimiento curricular, así como su representación, sigue siendo un elemento de debate.

\section{OBJETO DE LA INVESTIGACIÓN}

El objetivo del presente trabajo fue caracterizar desde el CDC la componente del conocimiento curricular para una profesora de física de Bachillerato en Colombia durante dos años consecutivos, antes y después de un programa de intervención. Este objetivo se plasma en las siguientes preguntas:

1. ¿Cuál es el conocimiento que se tiene sobre el currículo a nivel declarativo, de diseño y acción durante la enseñanza del campo eléctrico?,

2. ¿Cuáles son los obstáculos, o elementos facilitadores, que le permiten al profesor realizar modificaciones sobre su conocimiento curricular para la enseñanza del campo eléctrico?

\section{METODOLOGÍA}

La investigación se desarrolló con una profesora licenciada en física con formación en didáctica de las ciencias, de 28 años y una experiencia docente de 7 años, que desarrolla su labor en una institución educativa de carácter privado en la ciudad de Bogotá, Colombia. Sus estudiantes tienen edades que oscilan entre los 17 y 19 años.

Un multimétodo de evaluación (BAXTER; LEDERMAN, 1999) fue utilizado para caracterizar el conocimiento sobre el currículo, que incluye: a) un cuestionario de preguntas abiertas sobre lo que la profesora considera que son las estrategias de enseñanza en física y el papel de la planificación en el proceso de enseñanza-aprendizaje; b) el material curricular utilizado por la profesora; c) la plantilla dispuesta por Pro (1998) para realizar planificaciones; d) la matriz diseñada por Loughran, Mulhall y Berry (2004) como representación del contenido (ReCo), a la que se le han realizado algunas modificaciones en cuanto al número de preguntas, e) entrevista semi-estructurada y f) observaciones de clase.

La sistematización de los datos y su análisis se realizó siguiendo las técnicas de análisis de contenido (BARDIN, 1986; FRAENKEL; WALLEN, 2009), que incluye 
los siguientes pasos: a) identificación de las unidades, información procedente de cada instrumento o herramienta utilizada para recolectar los datos, atendiendo al contenido manifiesto; b) codificación de las unidades de información en las categorías y subcategorías; c) categorización de las unidades de información; d) análisis de las unidades de información; e) incorporación de categorías emergentes a la descripción a partir de los análisis realizados (SOLÍS et al., 2012).

Se utilizó en el análisis un sistema de categorías mixto en el cual se consideraron las categorías utilizadas en las investigaciones sobre el CDC en física y las emergentes durante el proceso de análisis. Los ámbitos analizados fueron: (B1) contendidos a enseñar, y/o patrón temático; (B2) organización y relación entre contenidos; (B3) criterios de selección de los contenidos; (B4) importancia del contenido a enseñar; (B5) objetivos de aprendizaje y enseñanza; (B6) fuentes de información y recursos utilizados por el profesor. Para apoyar el proceso de codificación se utilizó el software NVivo 10.

Para la descripción de cada categoría, se tomó en cuenta las pruebas aportadas por la información analizada, las investigaciones sobre el CDC en física y por los modelos didácticos en enseñanza de la ciencia (MELO et al., 2018). Para describir cada categoría adoptamos dos orientaciones básicas (la tradicional centrada en el profesor, y la alternativa centrada en los estudiantes) y una intermedia (MELO et al., 2018). En el anexo 1, se muestra el sistema de categorización utilizado. Además de la organización por tendencias, el análisis se subdividió según lo que la profesora declara o piensa (nivel declarativo), lo que dice que hará (nivel diseño) y lo que hace durante la enseñanza del campo eléctrico (nivel de acción).

El análisis del nivel declarativo (A.1) es el resultado de la triangulación de tres instrumentos: a) la entrevista semi-estructurada realizada a la profesora al iniciar su curso, b) la matriz representación del contenido (ReCo), y c) el cuestionario cuya intención principal fue reforzar algunas de las respuestas dadas por la profesora sobre la enseñanza del campo eléctrico, la planificación y las estrategias de enseñanza. El nivel de diseño (A.2) es el resultado de la triangulación de a) la unidad didáctica diseñada, b) los apuntes de clase preparados por la profesora y c) la programación anual. El nivel de acción (A.3), en esencia lo conforman las observaciones de las clases, apoyado por el ReCo y las entrevistas.

Para confirmar la fiabilidad de los resultados se llevaron a cabo varias rondas de análisis. Los resultados fueron validados por tres investigadores en didáctica de las ciencias. Además, los análisis fueron compartidos y revisados por la profesora. Se consiguieron acuerdos del 92\% (los desacuerdos se resolvieron mediante consenso).

\section{LA INTERVENCIÓN}

La fase de intervención se efectuó al finalizar las entrevistas y después de realizar un primer análisis global de los instrumentos de recolección de datos. El objetivo fundamental de la intervención fue el desarrollo de una nueva unidad didáctica sobre el campo eléctrico, tomando como referencia el análisis y la reflexión de las propias conductas de aula, así como lo que piensa y planifica 
la profesora. Asumimos que los procesos de intervención con el profesorado deben basarse en la reflexión continua, autorregulada y metacognitiva, a partir de su contexto de trabajo cotidiano.

La decisión de los aspectos trabajados se consensuó con la profesora según sus intereses desde tres fuentes: a) las reflexiones teóricas sobre la enseñanza del campo y la fuerza eléctrica y las componentes del CDC; b) los intereses y dificultades de la profesora sobre la enseñanza del campo y la fuerza eléctrica; y c) el análisis global de los datos, los cuales nos permitieron idear un perfil general de su CDC. La intervención finalmente se centró en tres aspectos: i) reuniones formativas, ii) análisis con la profesora de la observación de las clases, la planificación, el ReCo, y la entrevista, iii) la preparación de la nueva unidad didáctica.

\section{RESULTADOS}

La presentación de los resultados se dividirá en dos grandes apartados, los cuales contribuyen a dar respuesta al objetivo de la investigación. Por un lado, presentaremos el análisis cualitativo y por otro el cuantitativo. En el análisis cuantitativo, presentaremos nuestros aportes sobre la forma de representar la dimensión curricular de la profesora participante. Para presentar los resultados describiremos primero las características de la dimensión curricular, antes y después de la intervención. Finalizaremos describiendo los factores del cambio.

\section{ANÁLISIS CUALITATIVO}

Los resultados se presentan aludiendo a cada uno de los niveles analizados: declarativo, diseño y acción. Para cada uno de ellos se ha realizado una descripción general de la tendencia tradicional o centrada en el profesor, intermedia y alternativa antes y después de la intervención.

\section{Nivel Declarativo}

\section{Antes de la Intervención}

La mayor parte de las unidades reflejan una tendencia tradicional. La tendencia tradicional está mediada por la secuencia de contenidos que dispone la profesora para la unidad de electricidad, donde se parte de lo simple, los fenómenos triboeléctricos a través de una secuencia lineal, hasta llegar a la idea de campo y su superposición (B1).

La carga eléctrica es considerada el concepto más sencillo de aprender; además es el más utilizado a lo largo de la secuencia de contenidos propuesta (B2). Sin embargo, la profesora expresa dudas durante la entrevista sobre la eficacia de la ruta temática planteada, debido al ejercicio de reflexión que le supuso plasmar su enseñanza a través del ReCo (B3). 
eléctrico y campo magnético me van a entender las ondas electromagnéticas y en acústica puedo hacerlo breve, además que mira que como, como propusimos el programa con Esperanza, las niñas bueno no sé, es que eso también habría que pensarlo, porque ellas en noveno están viendo pinitos de electricidad y magnetismo entonces digamos que hasta ahora de pronto mirando con esos grupos que vienen podríamos continuar con lo que tenemos propuesto hasta ahora [Entrevista antes de la intervención, B3-1]

Forman parte de esta tendencia tradicional por un lado el tiempo, como recurso que regula el patrón temático organizado para la comprensión del contenido campo y, por otra parte, los objetivos trazados por los exámenes estandarizados externos (B6). En términos generales, la profesora considera que se puede prescindir de la idea de campo eléctrico en el programa de bachillerato, tal como se hace en secundaria, si se piensa en la enseñanza de los circuitos (B1). También comenta que la idea de campo eléctrico se introduce en su currículo “por su propia satisfacción personal” (B2).

La profesora indica que sus experiencias universitarias juegan un papel importante en la determinación de los recursos que utiliza en el aula sobre los fenómenos electrostáticos. Prácticamente los considera una réplica (B5). También el uso de la visión de la historia durante su formación universitaria, aspectos que hemos clasificado en la tendencia intermedia. La profesora considera que, aunque el profesor no incluya estas reflexiones en el aula, sí son necesarias en la recontextualización del saber que el profesor realiza sobre los conceptos que enseña.

Para finalizar el análisis del primer año, describiremos el resto de las unidades clasificadas en la tendencia alternativa. La profesora menciona que, para ella, es importante que los programas de secundaria y bachillerato estén articulados (B3), para que las estudiantes perciban un conocimiento físico que se interrelaciona. Con relación al material didáctico que utiliza para la clase, lo describe como flexible, pensado para dar respuesta a los objetivos y para ser útil a las estudiantes (B5). A lo largo de la entrevista la profesora expresa su inconformidad con el uso de un único libro de texto y reclama la autonomía que tiene en secundaria para desarrollar su propio material (B5).

\section{Después de la intervención}

Durante el desarrollo de la entrevista del segundo año, la profesora reconoce que la organización de los contenidos (B2) propuesta, “se guía más por el libro de texto que por la estructura lógica de la disciplina, principalmente en cuanto a la relación entre la visión de la luz como onda y el campo electromagnético”. La profesora considera que una estructura coherente con la física misma vincularía primero el capítulo de electricidad y magnetismo y luego el de ondas y óptica. Sin embargo, acciones externas como el examen de selectividad o los contenidos programados en secundaria hacen que la profesora mantenga una mirada conservadora hacia la estructura de los contenidos (B3). 
La profesora sigue considerando que a este nivel de escolaridad "se puede prescindir de la idea de campo, y mantiene la ruta que va de la carga al campo" (B1, B2). Sin embargo, la profesora incluye la fuerza después de la idea de campo, y antes de definir su intensidad. Esta modificación fue posible gracias al énfasis que la profesora asigna a la interacción, como efecto del campo y no como único producto de las interacciones de dos cargas (B3). Además, está convencida de la necesidad de relacionar lo electrostático y lo electrocinético (B2), situación que no era contemplada el año anterior. Pensando en términos del aprendizaje de sus estudiantes, insiste en que "el análisis del comportamiento de los imanes es la ruta de entrada más efectiva hacia la idea de campo”, que aquella donde se parte de los fenómenos de electrificación (B1, B3).

Después de la intervención, la profesora reconoce que las expectativas sobre el aprendizaje de los contenidos están en función del nivel de abstracción que el profesor asigna al contenido (B3). Para la profesora el nivel de abstracción no sólo depende de la estructura sintáctica de la teoría, sino del tipo de estrategias disponibles para su enseñanza y las comprensiones que el profesor tiene sobre el contenido.

En términos de las tendencias tradicional, intermedia y alternativa, la profesora desde su discurso relata cómo su comprensión del currículo cambia de acuerdo con los intereses y necesidades de sus estudiantes, y como intenta presentar, a través de su discurso, ejemplos o situaciones donde se relacione la temática con el contexto cotidiano o el futuro universitario de las estudiantes (B2, B3). Además, cambia los objetivos descritos incluyendo los referidos a "promover la conciencia, uso y reciclaje de aparatos electrónicos, y la energía eléctrica” (B6).

\section{Nivel Diseño}

\section{Antes de la Intervención}

La profesora en sus planificaciones se posiciona en una idea de carga desde el modelo atómico. Sin embargo, las partículas elementales no siempre son utilizadas para describir lo que significa estar cargado (B1). Para ser la carga uno de los contenidos principales, como cita la profesora desde sus declaraciones antes de la intervención, tiene poco protagonismo en los inicios del desarrollo de la unidad didáctica de electricidad y magnetismo.

El patrón temático (B1) que mantiene la profesora es igual al declarado. Para la profesora, el primer acercamiento al comportamiento eléctrico de la materia se centra en las fuerzas y no en la presencia del campo eléctrico (B1, B2). La descripción de dichos contenidos se presenta de manera secuenciada, y el tipo de relaciones causales entre fuerza y campo sólo se enuncia como objetivo, el cual no es fácil de rastrear dentro de su planificación (B3). El resto de los objetivos explícitos formulados en algunas ocasiones no corresponden a los descritos en el programa o la rúbrica de evaluación (B6).

Nuevamente, la tendencia tradicional mantiene una mayor representación en concordancia con lo declarado, aunque hay evidencia del resto de tendencias. Forman parte de la tendencia tradicional la descripción realizada sobre los contenidos, la cual se centra en la presentación de los contenidos siguiendo 
una secuencia lineal, de lo más simple a lo más complejo, tal como declara (B1). Además, la participación que se les da a los estudiantes durante su planificación es muy poca.

La tendencia intermedia se caracteriza por la referencia tanto a contenidos conceptuales como procedimentales (B6). En los contenidos conceptuales se favorecen la comprensión y en los procedimentales, el aplicar los contenidos explicados. Ambos descriptores pertenecen, según la categorización de Bloom (KRATHWOHL, 2002), a niveles cognitivos bajos. Al comparar los objetivos que la profesora piensa que sus estudiantes deben alcanzar, con los que planifica en su unidad didáctica y los acordados institucionalmente, vemos que los propuestos institucionalmente buscan niveles cognitivos altos, a diferencia de lo planificado. Esto está en concordancia con procesos de aprendizaje tendientes hacia la asimilación de la información.

En la tendencia alternativa hemos clasificado las descripciones que dan cuenta de los aspectos históricos, que se consideran como apoyo a las explicaciones sobre la electrificación, y la relación sucinta entre la electrostática y electrocinética que, aunque se menciona, no se desarrolló durante la unidad didáctica (B2). También forman parte de esta tendencia el uso de las experiencias demostrativas en clase relacionadas con la electrificación de los cuerpos, el uso del generador de Van der Graaf, y también las actividades de motivación (B5).

\begin{abstract}
Por medio de un montaje sencillo, con globos, una barra de papel aluminio suspendido de nylon, barras da plástico, lana, barras de vidrio, seda y un electroscopio se pretende que los estudiantes observen cuál es el comportamiento de los cuerpos luego de ser frotados con uno u otro material y en qué casos se presentan fuerzas de atracción o repulsión. [...] Toma mucha importancia al permitirle al estudiante OBSERVAR lo que la teoría plantea y captar su atención motivándolo hacia el tema. (Unidad Didáctica antes de la intervención, Sesión 2, Actividad de Laboratorio, B5-3)
\end{abstract}

\title{
Después de la Intervención
}

La planificación después de la intervención presenta un mayor nivel de desarrollo, y por tanto permitió rastrear unidades que el año anterior eran inexistentes. La modificación realizada el segundo año durante la fase de diseño, tiene que ver con la organización de los contenidos y la actividad inicial de la unidad didáctica (B2), la cual incluye la detección de las ideas previas de los estudiantes sobre la electrificación y la idea de campo.

En grupos (de 5 estudiantes) y con el material dispuesto para tal fin, las estudiantes seguirán instrucciones para desarrollar experiencias diferentes de electrostática, electrocinética y magnetismo, describirán lo que observan y escribirán una explicación para lo que sucede. [...] La intención es que las 
estudiantes con sus conocimientos previos asociados tanto con sus cursos previos de física, como con su experiencia cotidiana, logren explicar fenómenos de origen eléctrico y magnético y por medio de dichas explicaciones identificar posibles confusiones y o aciertos en el uso de dichos conocimientos. (Unidad Didáctica después de la intervención, Sesión 1, Actividad de Laboratorio, B2-3)

Desde la planificación, primero se plantea realizar un acercamiento hacia las interacciones eléctricas; para ello se utilizan las líneas de fuerza entre imanes, y el experimento de Oester. Desde estas experiencias se explica lo que es el campo magnético y por analogía, se presenta el campo eléctrico. Posterior a esta presentación, se formaliza el concepto introduciendo simbólicamente la expresión de la intensidad del campo eléctrico (B1).

En la nueva organización de los contenidos, la profesora intenta relacionar la parte de los circuitos y el magnetismo con la electrostática. También dota al campo magnético de energía, pero no al eléctrico. Esta organización refuerza la idea de la fuerza como efecto del campo, que trae grandes repercusiones en la organización y selección de las actividades (B2).

Como se describió en la fase declarativa, la profesora sigue visualizando al contenido no como un elemento estructurador del currículo, sino como aquel que sirve de requisito para el aprendizaje de otros contenidos de mayor nivel de abstracción, que no serán tratados en bachillerato. Esta asignación hace que el contenido sea considerado de poca relevancia para el aprendizaje de sus estudiantes (B1).

Aspectos como los roles de los estudiantes, la evaluación o la intención de presentar los contenidos en contexto, se siguen manteniendo. También se mantiene la presentación de los apartados históricos sobre la electrificación (B1). En términos generales, las intenciones frente al rol de los estudiantes y el profesor se mantienen. La profesora sigue siendo la fuente fundamental de conocimiento en el aula (B5). En términos de las unidades clasificadas en cada categoría, identificamos un descenso en la tendencia tradicional para el nivel de diseño, y un aumento de la tendencia intermedia y la tendencia alternativa.

\section{Nivel de Acción}

\section{Antes de la Intervención}

La información que aquí se presenta y analiza proviene de la transcripción de las observaciones de las clases, la entrevista, y el documento institucional que la profesora cumplimenta una vez finaliza sus clases.

De forma explícita no se proponen contenidos actitudinales, fuera de los que acontecen de forma espontánea durante las clases (B1). Para describir el concepto de carga eléctrica, la profesora hace referencia a situaciones anteriores, trabajadas durante el capítulo de óptica y la caracterización de la luz como onda. También reclama las imágenes que las estudiantes tienen sobre la estructura y algunas propiedades de la materia, vistas en sus cursos de química, ya que le permiten 
situar el modelo de carga a nivel atómico (B1, B3). El contenido de carga es tal vez la única oportunidad explícita de vincular situaciones cotidianas a su clase.

En cuanto a la fuerza eléctrica, son sólo dos las menciones que la profesora realiza sobre el papel del medio en la descripción de la fuerza eléctrica, frente a las 77 intervenciones durante las cuales la fuerza es descrita sólo como fuerza entre cargas. En dos ocasiones hace alusión a la necesidad de introducir un nuevo marco de referencia, la teoría de campo para describir lo que acontece. Sin embargo, no muestra la relación con los desarrollos y experimentos de Coulomb en la determinación de su ley. En ocasiones los procedimientos no son considerados como contenidos sino como parte de la metodología para lograr la adquisición conceptual, como expresa a continuación:

\footnotetext{
[...] y si yo quiero calcular el efecto debido a varias fuerzas eléctricas, pues tendré que sumarlas vectorialmente. Su combinación se encuentra entonces, hallando cada fuerza por separado y sumándolas vectorialmente, y..., pues, regularmente va a ser una disposición geométrica de éstas; cuando yo digo geométrica, implica que estén ubicadas de manera que yo pueda medir la distancia que las separa, porque si yo voy a calcular una fuerza eléctrica necesito de esa distancia además de las magnitudes de las que aquí tengo dispuestas [Clase 2].
}

La articulación de los contenidos se presenta de forma secuencial. La profesora enumera, a medida que se desarrolla la clase, los contenidos explicados (B2). De forma organizada sitúa el título del concepto que explica, luego el ejemplo de apoyo utilizado para explicar el concepto, con su respectiva representación anota la definición, y procede a plantear un ejercicio que implique la realización de cálculos (B3).

Como se muestra en la Gráfica 1, las ideas que expresan los estudiantes sobre los fenómenos electrostáticos propuestos son fuentes de información primarias; también lo es, mayoritariamente, la profesora a través de su discurso y las distintas estrategias que utiliza para presentar la información (B5), independientemente del contenido que se enseñe.

La mayoría de las intervenciones de los alumnos, en las que aportan información, son por requerimiento de la profesora. Menos frecuentes son las intervenciones donde las estudiantes aportan información por iniciativa propia. Se debe a las rutinas y criterios de validez y autoridad establecidos en el aula. La profesora, el experimento, el científico o la teoría son los que al fin de cuentas tiene la última palabra sobre lo que se dice en el aula (B3). Encontramos que se utiliza poco el libro de texto como fuente de información, aunque sigue su secuencia de contenidos.

Para ilustrar la información, la profesora utiliza distintos medios. Hace mención de diferentes representaciones gráficas e imágenes sobre el campo y el desarrollo de una experiencia demostrativa sobre las líneas de fuerza. También incluye la visualización de videos como apoyo a las experiencias demostrativas, 
para que las estudiantes puedan observar en detalle lo que la profesora les quiere mostrar. El uso de la pizarra es el recurso más utilizado. Esto apoya una enseñanza centrada en el profesor.

En resumen, la tendencia a nivel curricular es tradicional con matices en la tendencia alternativa. La tendencia tradicional se caracteriza por enseñar mayoritariamente contenidos conceptuales. Utilizar en algunas ocasiones hechos de la vida cotidiana, pero como ejemplos. Además, es la profesora quien aporta la mayor parte de la información, a través de explicaciones y preguntas, aunque haga uso de otras fuentes de información. La tendencia alternativa es definida por la inclusión de hechos de la vida cotidiana, la historia de la ciencia y la utilidad dada a las experiencias demostrativas como contenido y apoyo a las explicaciones.

\section{GRÁFICA 1}

FUENTE DE INFORMACIÓN EN EL AULA ANTES DE LA INTERVENCIÓN DESDE EL NIVEL DE ACCIÓN

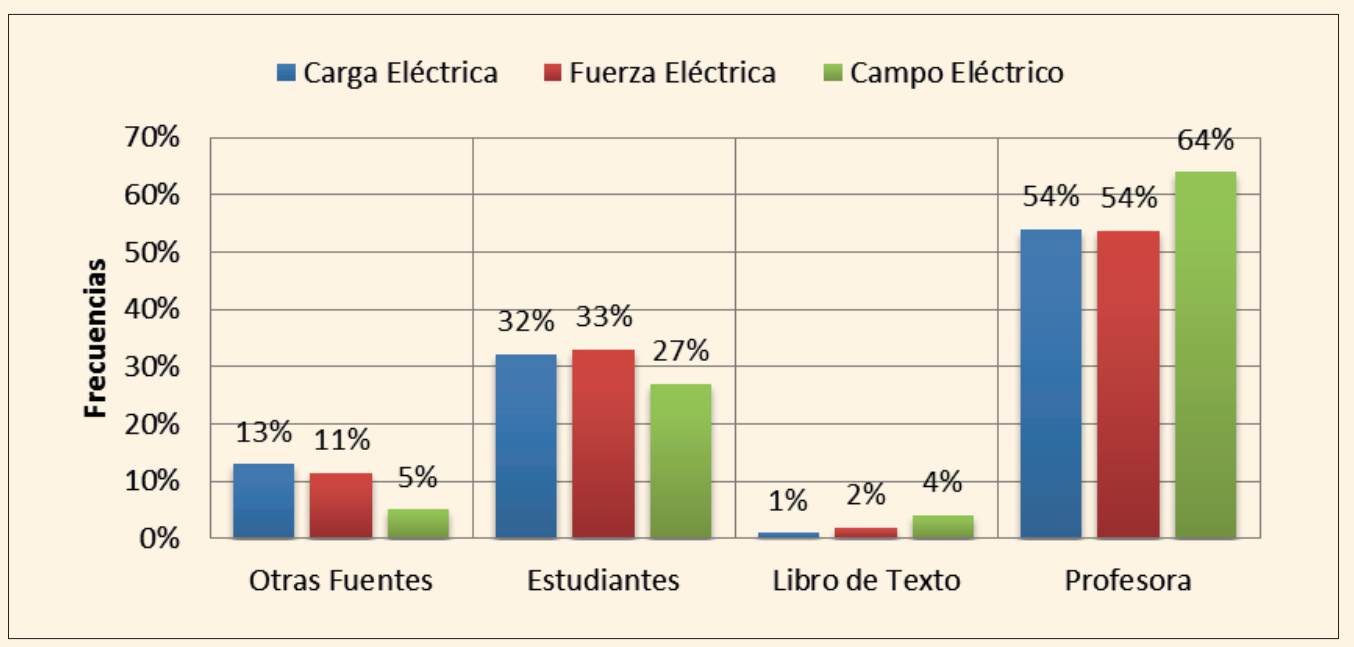

Fuente: Elaboración propia.

\section{Después de la Intervención}

La profesora considera que la comunidad educativa ha realizado un énfasis excesivo en considerar los exámenes de selectividad como medida de eficacia del profesor. Dicho énfasis ha influido en sus objetivos de enseñanza para bachillerato en comparación con los de secundaria (B3). La profesora menciona, durante la reflexión, que se asume una perspectiva más del contenido por el contenido, enmascarando ejemplos con hechos cotidianos en su hacer en el aula (B1). Esto hace que ella reemplace el tiempo de debate y la discusión por clases expositivas, ya que es la metodología que permite cubrir más contenidos en un menor tiempo (B2).

La profesora parte de una postura donde las cargas son el principio organizador del patrón temático a una donde son los efectos del campo, tal como se concluyó en el nivel de diseño. La presentación de los contenidos en la acción sigue evidenciando una visión acumulativa y lineal cuando el campo eléctrico se relaciona con la fuerza para explicar las ecuaciones que describen la intensidad del campo. 
Notamos que la profesora hace mayor énfasis en el concepto de conductor, en la distinción entre dieléctricos y conductores, y dedica menos refuerzo y tiempo a contenidos como polarización y electrificación por inducción (B1). También incrementa la cantidad de intervenciones sobre la fuerza eléctrica y la utilidad de la ley de Coulomb como heurístico para hallar el valor de la interacción entre cargas, o entre cargas y el campo eléctrico. Por otro lado, disminuye el énfasis dado a los datos y explicaciones históricas vinculadas a la ley de Coulomb y el giro ontológico dado en el pensamiento físico al pasar de una concepción de fuerza a distancia a fuerza contigua (B3).

La profesora después de la intervención hace especial énfasis en la representación del campo, en términos de las líneas de fuerza y su representación vectorial. La intención de establecer relación entre la electrostática y los circuitos le lleva a mencionar o considerar más veces conceptos como energía, diferencia de potencial y corriente (B1). Además, la analogía que más se utiliza es aquella entre el campo magnético y el eléctrico.

\footnotetext{
Esas líneas de campo le dan a él (refiriéndose a Faraday) la idea de pensar en eso alrededor que llamó más adelante campo magnético, y nos permite entender muchas cosas en términos de la interacción. [Clase 2]
}

Como representamos en la gráfica 2, la profesora sigue siendo la fuente de información preponderante durante las clases que tratan contenidos como la fuerza y el campo eléctrico, y en un 50\% menos lo son las estudiantes. Respecto a la gráfica 1, la participación de las estudiantes aumenta considerablemente, sobre todo respecto a la carga eléctrica. No obstante, la mayoría de las intervenciones de las estudiantes son por requerimiento de la profesora. Las experiencias demostrativas, como fuente de información en el aula, son el principal factor que incluye nuevas dinámicas para la relación conocimiento-profesor-estudiante. Las diferentes expectativas hacia el logro sobre los objetivos de aprendizaje esperados, condicionan los énfasis dados a los contenidos procedimentales.

El nivel de acción en término de las tendencias presenta un leve incremento en la tendencia intermedia y la tendencia alternativa, y una disminución en la tendencia tradicional. Sin embargo, en términos globales, sigue primando para el nivel de acción la tendencia tradicional e intermedia. 


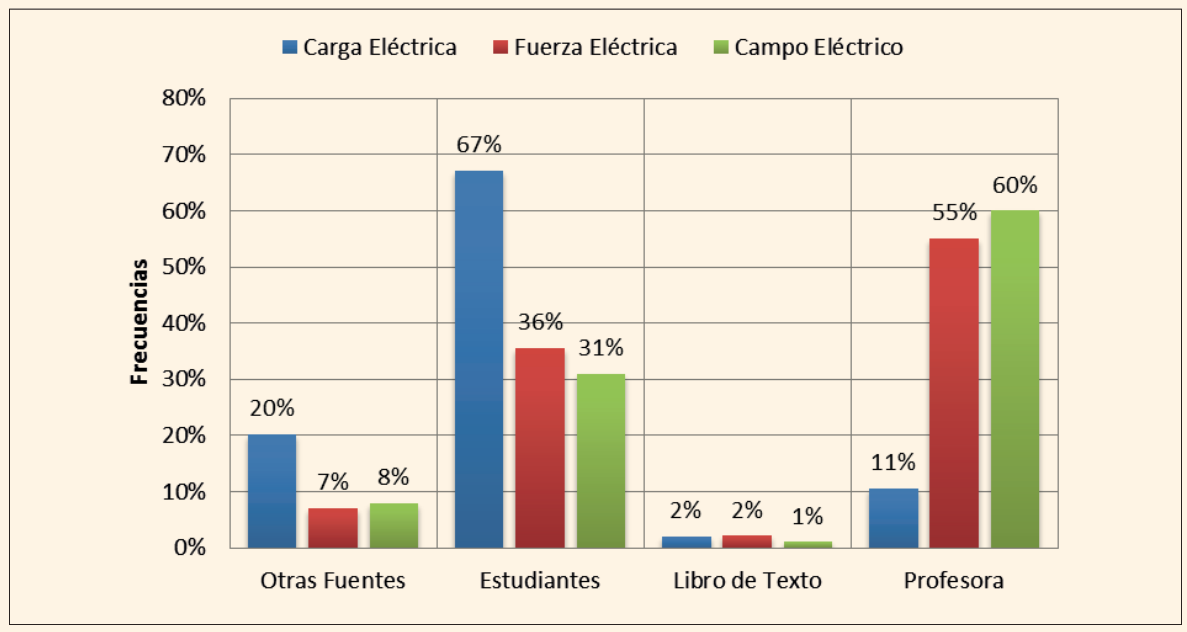

Fuente: Elaboración propia.

\section{ANÁLISIS DESCRIPTIVO: UNA FORMA DE REPRESENTAR LA DIMENSIÓN CURRICULAR}

Una vez analizado cada instrumento y recolectadas todas las unidades para una misma tendencia según lo que la profesora declara, diseña y hace, se construyeron las gráficas 3 y 4. Este instrumento nos indica el énfasis que la profesora realiza sobre cada nivel analizado. En azul simbolizamos la tendencia tradicional, en rojo la intermedia y en verde la alternativa.

Las gráficas como instrumento de análisis nos permiten estudiar los cambios y transiciones para cada nivel analizado, y el modelo de enseñanza-aprendizaje que la profesora utiliza desde lo que piensa, planifica y hace en el aula. Antes de la intervención vemos cómo la tendencia tradicional prima mayoritariamente en cada uno de los niveles, ratificando una gran coherencia de su conocimiento curricular entre lo que la profesora declara, planifica y hace en su aula. Después de la intervención, se nota grandes modificaciones en cuanto a lo que declara, incrementándose las unidades clasificadas en la tendencia intermedia y alternativa, coexistiendo así en proporciones comparables dichas tendencias. El nivel de diseño es aquel que mayores cambios presenta, notándose principalmente un incremento considerable en la tendencia intermedia; esto se debe a la integración del ejercicio de diseño en el programa de intervención. Estos resultados muestran la importancia del diseño curricular de la enseñanza en los procesos de concreción del CDC.

Consideramos que los procesos de diseño curricular mentales conforman un primer estado de concreción del CDC. El desarrollo de las unidades didácticas como actividad de reflexión desde y sobre el trabajo del aula, conformaría un nivel superior de esa concreción porque

[...] cuando un profesor planifica una unidad didáctica, una lección o unas actividades, integra sus conocimientos científicos y didácticos, su experiencia práctica y sus 
concepciones ideológicas, lo que no suele suceder cuando "se copia la programación del año anterior". (DE PRO, 1999, p. 411)

GRÁFICA 3

CONOCIMIENTO CURRICULAR ANTES DE LA INTERVENCIÓN

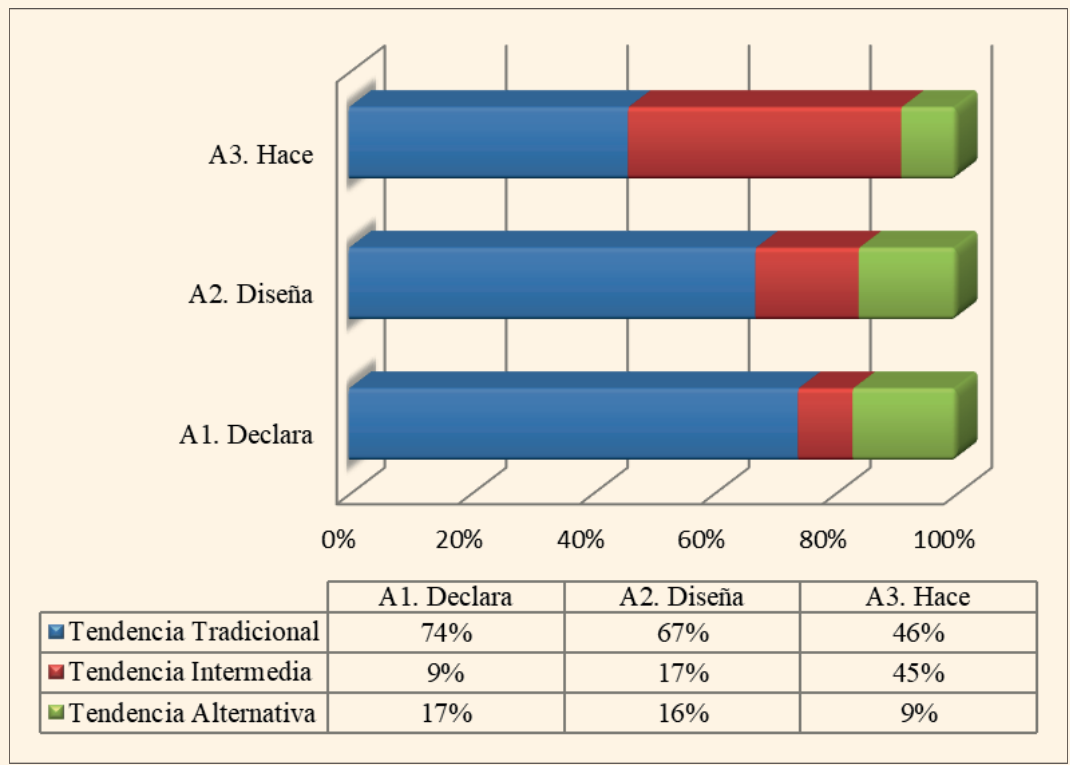

Fuente: Elaboración propia.

GRÁFICA 4

CONOCIMIENTO CURRICULAR DESPUÉS DE LA INTERVENCIÓN

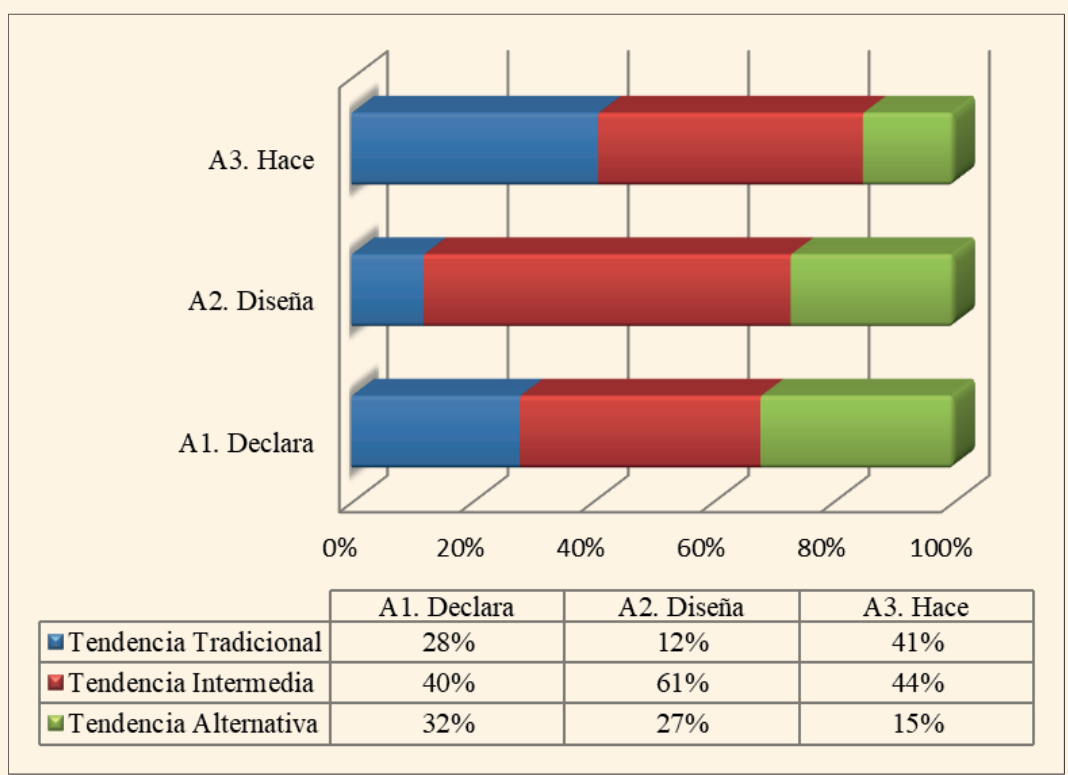

Fuente: Elaboración propia.

Otro instrumento que desarrollamos para dar cuenta del conocimiento curricular, a partir del análisis de las frecuencias de cada categoría, se resume en la tabla 2. Una vez identificadas la cantidad de unidades por tendencia, seleccionamos la que mayor representación mantenía en comparación con las 
demás. Este instrumento nos permite estudiar los cambios y transiciones de cada categoría en mayor profundidad.

Hemos sombreado con gris claro aquellas categorías que han cambiado de una tendencia tradicional (TT) a una intermedia (TI), de una tradicional a una mezcla de todas las tendencias, o de una mezcla de tendencias a una intermedia. En gris oscuro se resaltan las categorías que muestran cambios de una mezcla de tendencias o una tendencia intermedia a una tradicional y de una tendencia alternativa a una tradicional. La mezcla de tendencias hace referencia a una distribución similar entre las frecuencias asignadas a la tendencia tradicional, intermedia o alternativa (TC), o con variaciones entre 0 a 5\%. También hemos incluido los casos en los cuales existe una distribución similar de frecuencias entre dos de las tendencias. Es decir, no hay una tendencia definida.

Para el caso de la profesora participante, la categoría referida a las fuentes y recursos que utiliza en el aula es aquella que no presenta modificación. La profesora reconoce el papel del estudiante como fuente de información en el aula, lo integra en su planificación, pero en la acción es ella la que adquiere el protagonismo, tal como se explicitó en el análisis cualitativo.

La profesora justifica estos resultados indicando su falta de conocimiento sobre las dificultades de los estudiantes. Este aspecto hace que durante las clases pase por alto situaciones de interés cognitivo. También reconoce que muchas veces "termina cayendo en el ejercicio de sustitución del pensamiento del estudiante por el pensamiento científico". Además, señala que la forma predominante en que los estudiantes asumen su aprendizaje, no le permite generar estrategias innovadoras centradas en los estudiantes.

TABLA 1

FRECUENCIAS REPRESENTATIVAS SOBRE EL CONOCIMIENTO CURRICULAR DURANTE LA ENSEÑANZA DEL CAMPO ELÉCTRICO ANTES Y DESPUÉS DE LA INTERVENCIÓN

\begin{tabular}{|c|c|c|c|c|c|c|}
\hline \multirow{2}{*}{ CATEGORÍAS } & \multicolumn{2}{|c|}{ A.1 DECLARATIVO } & \multicolumn{2}{|c|}{ A.2 DISEÑO } & \multicolumn{2}{|c|}{ A.3 ACCIÓN } \\
\hline & A-I & $\mathrm{D}-\mathrm{I}$ & A-I & D-I & A-I & D-I \\
\hline B1. Contenidos a enseñar y patrón temático & TT & TT & TT & TT & TT & TT \\
\hline B2. Criterios de selección de los contenidos & TT & O & TT & $\mathrm{O}$ & TT & TT \\
\hline B3. Organización y Relación entre contenidos & TT & $\mathrm{O}$ & TT & $\mathrm{O}$ & $\mathrm{O}$ & $\mathrm{O}$ \\
\hline B4. Importancia del contenido & TT & $\mathrm{O}$ & TT & $\mathrm{TI}$ & $\mathrm{TI}$ & $\mathrm{TI}$ \\
\hline B5. Fuentes y Recursos & $\mathrm{TI}$ & $\mathrm{TI}$ & $\mathrm{O}$ & $\mathrm{O}$ & TT & TT \\
\hline B6. Objetivos de Aprendizaje & TC & $\mathrm{TI}$ & TT & $\mathrm{O}$ & TT & TT \\
\hline
\end{tabular}

La otra categoría que no muestra cambios es la relacionada con el contenido a enseñar y el patrón temático. La profesora considera que el incumplir en la presentación de un contenido repercute en una alta probabilidad de fracaso en las respuestas a los exámenes de selectividad sobre el contenido de fuerza y campo eléctrico; por este motivo cambia poco la cantidad de los contenidos de un año a otro. La profesora reconoce también que sus creencias de eficacia hacia el contenido que enseña es otra razón que influye en la estructura de los contenidos. Al respecto menciona: 
[...] pues yo procuro llevar al aula las ideas con las que me siento cómoda y que siento que la claridad con la que cuento va a ser suficiente para no enredarlas a ellas, sé que quedan por fuera muchas otras pero son precisamente aquellas cosas en las que no me siento tan cómoda no les encuentro tanta importancia, pero aparte de encontrarles tanta importancia siento que no las entiendo muy bien, entonces no me arriesgo a llevarlas al aula como por ejemplo lo de la densidad de carga, como el potencial puede ser explicado ahí, para mí potencial, la capacidad que tiene el campo eléctrico de darle energía a la carga y cómo esa diferencia de potencial es la que permite que esa carga se mueva y produzca corriente eléctrica, hasta ahí. [Entrevista]

Frente a la permanencia en las tendencias en el nivel de acción, la profesora reconoce que durante la implementación de un nuevo contenido suele partir de una "enseñanza tradicional", es decir centrada en la exposición y ambientada con experiencias demostrativas para facilitar la asimilación de los contenidos, más que desde aproximaciones fenomenológicas; como declara, es su enseñanza en otros contenidos de su programa. Señala que el grado de frustración que experimenta durante su hacer en el aula, cuando las actividades planificadas no cumplen las expectativas deseadas, la lleva a retornar nuevamente a estrategias expositivas. Al respecto dice:

[...] porque para mí es más a gusto que, para mí personalmente es emocionante que vean, y esa misma emoción yo quiero despertarla en ellas, que ellas digan uy, sí eso es verdad, y no eso lo produjeron no sé yo en donde, ni quien, y yo [...] pues estoy viendo ahí el rayo, pero no me convence, y por eso trato de hacer el esfuerzo [...]

[...] obviamente cuando no funciona, pues yo induzco ante ellas la respuesta que quiero ante las preguntas como muestras ahí, pues porque no me puedo quedar ahí, la clase sigue, entonces el factor tiempo juega ahí un papel muy importante. [Sesión de Reflexión 7]

Finalmente, los cambios detectados por la profesora y reflejados en la tabla 2 se deben a la organización que realiza del contenido que enseña y a la flexibilidad que le aporta esta reestructuración sobre la selección y organización de su enseñanza, lo que a su vez repercute en las declaraciones que efectúa sobre el aprendizaje. Por otro lado, la involución en la parte declarativa muestra una perspectiva más consciente del trabajo que se hace durante la práctica con relación a la enseñanza del campo eléctrico. 


\section{CONCLUSIONES Y DISCUSIÓN}

La caracterización del CDC es un ejercicio complejo que requiere de mayor documentación y protagonismo en el caso de la enseñanza de física en secundaria y más aún en bachillerato. El presente artículo muestra de forma detallada cómo hacer explícito el conocimiento curricular para contenidos de física, y planteamos una forma de representarlo. Con relación a la primera pregunta relacionada con la caracterización del conocimiento curricular antes y después del programa de intervención, encontramos que muchos de los constructos, que a nivel declarativo la profesora ha recabado a lo largo de su experiencia, no se transponen de forma lineal a su práctica de aula.

Por otro lado, la intervención que se realiza con la profesora desestabiliza su alta coherencia frente a la tendencia tradicional en cada uno de los niveles considerados. Después de la intervención se notan grandes modificaciones en cuanto a lo que declara, incrementándose las unidades clasificadas en la tendencia intermedia y alternativa. Sin embargo, es el nivel de diseño el que representa los mayores cambios, notándose un incremento considerable en la tendencia intermedia, aunque la acción siga manteniendo una tendencia tradicional. Estos resultados no difieren de los propuestos por Solís, Porlán y Rivero (2012) con profesores de ciencia y nos sugieren la importancia de emprender más estudios focalizados en el CDC en la acción. Además, nos indican que es el CDC en la acción el más resistente al cambio, el más difícil de caracterizar y sobre el cual se debe enfocar con mayor insistencia los programas de intervención.

Para el caso de la profesora participante, la categoría referida a las fuentes y recursos que utiliza en el aula y la selección de los contenidos centrales para la enseñanza del campo eléctrico no presentan modificación en la tendencia predominante después de la intervención. Sin embargo, la comprensión que la profesora tiene del conocimiento del contenido ejerce una elevada influencia en el modo de secuenciar y presentar los diferentes temas y las dinámicas que utilizan en el aula, situación que concuerda con los estudios de Park y Oliver (2008) y Sadler et al. (2013).

El posicionamiento desde la tendencia tradicional frente a lo que la profesora declara, diseña y hace sobre los contenidos, reflejo de una secuencia de contenidos que inicia por la carga eléctrica y finaliza en la enseñanza de los circuitos, según Reyes y Martínez (2013), tiene de trasfondo una visión de campo eléctrico como instrumento para analizar la interacción desde una perspectiva en la que la diferencia entre fuerza y campo está ausente. La profesora compensa esta dificultad incluyendo en su discurso la idea de la fuerza como efecto del campo después de la intervención, y señalando la inclusión de ejemplos que parten de situaciones magnéticas más que mecánicas. La propuesta de Varela, Pérez y Manrique (2000), donde se utilizan como ejes curriculares las fuentes y efectos del campo y los principios de conservación de la carga, corriente y energía, aunque es considerada por la profesora no es desarrollada. Esto se debe a sus creencias de autoeficacia negativas hacia la comprensión del contenido que enseña y la poca importancia que le asigna a la enseñanza del campo eléctrico. 
En cuanto a los recursos y fuentes de conocimiento, los resultados nos sugieren que el conocimiento que la profesora tiene sobre los estudiantes impacta sobre el conocimiento en lo que se refiere a los recursos, situación que difiere de los estudios de Lee y Luft (2008) donde es el conocimiento sobre los recursos el que influye sobre la organización curricular, la selección de estrategias de enseñanza, y la utilización de las evaluaciones.

La imagen que observamos de la coexistencia de las tres tendencias - declarativa, diseño y acción -, lejos de ser contradictoria, da cuenta de la transición sobre la organización de la enseñanza del campo eléctrico a medida que integra y transforma su conocimiento sobre la disciplina, los estudiantes, la evaluación, las estrategias de enseñanza y las negociaciones que establece entre su idea de aprendizaje y enseñanza. Aunque consideramos que el cambio en cada tendencia es fundamental, más si es hacia la alternativa, un paso esencial en el proceso de sofisticación de esta componente del CDC es establecer coherencias entre lo que el profesor dice, diseña y hace.

Con relación a la segunda pregunta de nuestro estudio relacionada con los obstáculos o elementos facilitadores, uno de los mayores obstáculos y a la vez facilitadores del cambio, está relacionado con los conocimientos científicos, didácticos o prácticos que la profesora mantiene sobre la enseñanza del campo eléctrico, un factor que en repetidas ocasiones ha sido citado en la literatura (HALIM; MEERAH, 2002). Otros elementos relevantes son las emociones que la profesora experimenta hacia las estrategias que implementa, sus creencias de autoeficacia como ya se ha mencionado y la escasez de materiales curriculares adaptados a las imágenes de aprendizaje que elabora la profesora (MELLADO, 2001).

El estudio nos ha permitido poner en evidencia los elementos que permanecen sobre la dimensión curricular, y como otros van cambiando y son susceptibles de evolucionar. En concordancia con Vázquez, Jiménez y Mellado (2010) consideramos que los cambios del profesorado "son lentos y graduales, afectan más a unos ámbitos que a otros, síntoma de las interacciones múltiples que se establecen y de los diferentes condicionantes a que se ve sometida la profesora en todo el proceso" (p. 426).

Consideramos que la participación de la profesora en el programa de intervención ha conseguido implicarla en el desarrollo de su CDC. Además, el ejercicio de planificación y el diseño de la unidad didáctica fue imprescindible para comprobar y consolidar la consistencia de los cambios. Sin embargo, como señalan Vázquez, Jiménez y Mellado (2019) es necesario profundizar sobre la formación inicial del profesorado ya que es un elemento central para el desarrollo profesional.

\section{REFERENCIAS}

ABELL, S. K. Research on science teacher knowledge. In: ABELL, S.; LEDERMAN, N. (ed.). Handbook of research on science education. New York: Lawrence Erlbaum Associates, 2007, p. 1105-1149. 
BAXTER, J. A.; LEDERMAN, N. G. Assessment and measurement of pedagogical content knowledge. In: GESS-NEWSOME, J.; LEDERMAN, N. G. Examining pedagogical content knowledge: the construct and its Implications for science education. Dordrecht: Springer, 1999. p. 147-161.

CHEUNG, D.; WONG, H. W. Measuring teacher beliefs about alternative curriculum designs. Curriculum journal, v. 13, n. 2, p. 225-248, 2002.

CONTRERAS, S. A. Qué piensan los profesores sobre sus clases: estudio sobre las creencias curriculares y las creencias de actuación curricular. Formación Universitaria, v. 1, n. 3, p. 3-11, 2008.

CONTRERAS, S. A. Creencias curriculares y creencias de actuación curricular de los profesores de ciencias chilenos. Revista Electrónica de Enseñanza de las Ciencias, v. 8, n. 2, p. 505-526, 2009.

FRAENKEL, J.; WALLEN, N. How to design evaluate research in education. New York: Mc. GrawHill, 2009.

FRIEDRICHSEN, P. M.; DANA, T. M. Using a Card-Sorting Task to Elicit and Clarify Science. Journal of Science Teacher Education, v. 14, n. 4, p. 291-305, 2003.

FRIEDRICHSEN, P. M.; VAN DRIEL, J. H.; ABELL, S. K. Taking a closer look at science teaching orientations. Science Education, v. 95, n. 2, p. 358-376, 2011.

FRIEDRICHSEN, P. M.; ABELL, S. K.; PAREJA, E. M.; BROWN, P. L.; LANKFORD, D. M.; VOLKMANN, M. J. Does teaching experience matter? Examining biology teachers' prior knowledge for teaching in an Alternative Certification Program. Journal of Research in Science Teaching, v. 46, n. 4, p. 357-383, 2009.

HALIM, L.; MEERAH, S. M. Science trainee teachers' Pedagogical Content Knowledge and its Influence on physics teaching. Research in Science \& Technological Education, v. 20, n. 2, p. 215-225, 2002.

HENZE, I.; VAN DRIEL, J. H.; VERLOOP, N. Development of experienced science teachers' Pedagogical Content Knowledge of models of the solar system and the universe. International Journal of Science Education, v. 30, n. 10, p. 1321-1342, 2008.

JUSTI, R.; VAN DRIEL, J. The development of science teachers' knowledge on models and modelling: promoting, characterizing, and understanding the process. International Journal of Science Education, v. 27 , n. 5, p. 549-573, 2005.

KRATHWOHL, D. R. A revision of Bloom's taxonomy: an overview. Theory into practice, v. 41, n. 4, p. 212-218, 2002.

LEE, E.; LUFT, J. A. Experienced secondary science teachers' representation of pedagogical content knowledge. International Journal of Science Education, v. 30, n. 10, p. 1343-1363, 2008.

LOUGHRAN, J.; MULHALL, P.; BERRY, A. In search of pedagogical content knowledge in science: developing ways of articulating and documenting professional practice. Journal of Research in Science Teaching, v. 41, n. 4, p. 370-391, 2004.

MAGNUSSON, S.; KRAJCIK, L.; BORKO, H. Nature, sources and development of pedagogical content knowledge. In: GESS-NEWSOME, J.; LEDERMAN, N. G. (ed.). Examining pedagogical content knowledge. Dordrecht, The Netherlands: Kluwer, 1999. p. 95-132.

MELLADO, V. ¿Por qué a los profesores de ciencias nos cuesta tanto cambiar nuestras concepciones y modelos didácticos? Revista Interuniversitaria de Formación del Profesorado, n. 40, p. 17-30, 2001.

MELLADO, V.; BORRACHERO, A. B.; BRÍGIDO, M.; MELO, L.V.; DÁVILA, M. A.; CAÑADA, F.; CONDE, M. C.; COSTILLO, E.; CUBERO, J.; ESTEBAN, R.; MARTÍNEZ, G.; RUIZ, C.; SÁNCHEZ, J.; GARRITZ, A.; MELLADO, L.; VÁZQUEZ, B.; JIMÉNEZ, R.; BERMEJO, M. L. Las emociones en la enseñanza de las ciencias. Enseñanza de las Ciencias, v. 32, n. 3, p. 11-36, 2014.

MELO, L.; CARDONA, G.; CAÑADA, F.; MARTÍNEZ, G. Conocimiento didáctico del contenido sobre el principio de Arquímedes en un programa de formación de profesores de física en Colombia. Revista Mexicana de Investigación Educativa, v. 23, n. 76, p. 253-279, 2018. 
ORLEANS, A. V. Enhancing teacher competence through online training. The Asia-Pacific Education Researcher, v. 19, n. 3 , p. $371-386,2010$

PARK, S.; CHEN, Y.-C. Mapping out the integration of the components of Pedagogical Content Knowledge (PCK): examples from high school biology classrooms. Journal of Research in Science Teaching, v. 49, n. 7, p. 922-941, 2012.

PARK, S.; OLIVER, J. S. Revisiting the conceptualisation of Pedagogical Content Knowledge (PCK): PCK as a conceptual tool to understand teachers as professionals. Research in Science Education, v. 38, n. 3, p. 261-284, 2008.

PRO, A. Planificación de unidades didácticas por los profesores: análisis de tipos de actividades de enseñanza. Enseñanza de las Ciencias, v. 3, n. 17, p. 411-429, 1998.

REYES, J.; MARTÍNEZ, C. A. Conocimiento didáctico del contenido en la enseñanza del campo eléctrico. Tecné Episteme y Didaxis, TED, n. 33, p. 36-60, 2013

SADLER, P. M.; SONNERT, G.; COYLE, H. P.; COOK-SMITH, N.; MILLER, J. L. The influence of teachers' knowledge on student learning in middle school physical science classrooms. American Educational Research Journal, v. 50, n. 5, p. 1020-1049, 2013.

SCHNEIDER, R. M.; PLASMAN. K. Science teacher learning progressions: a review of science teachers' pedagogical content knowledge development. Review of Educational Research, v. 81, n. 4, p. 530-565, 2011.

SEUNG, E. The process of physics teaching Assistants' Pedagogical Content Knowledge Development. International Journal of Science and Mathematics Education, v. 11, n. 6, p. 1303-1326, 2013.

SHULMAN, L. Those who understand: knowledge growth in teaching. Educational Researcher, v. 15, n. 2 , p. $4-14,1986$.

SOLÍS, E.; PORLÁN, R.; RIVERO, A. ¿Cómo representar el conocimiento curricular de los profesores de ciencias y su evolución? Enseñanza de las Ciencias, v. 30, n. 3, p. 9-30, 2012

STENHOUSE, L. Investigación y desarrollo del currículum. Madrid: Morata, 1984.

VARELA, P.; PÉREZ, M. C.; MANRIQUE, M. Electricidad y magnetismo. Madrid: Síntesis Educación, 2000.

VÁZQUEZ-BERNAL, B.; JIMÉNEZ, R.; MELLADO, V. Los obstáculos para el desarrollo profesional de una profesora de enseñanza secundaria en ciencias experimentales. Enseñanza de las Ciencias, v. 28, n. 3, p. $417-432,2010$.

VÁZQUEZ-BERNAL, B.; JIMÉNEZ, R.; MELLADO, V. El conocimiento didáctico del contenido (CDC) de una profesora de ciencias: reflexión y acción como facilitadores del aprendizaje. Enseñanza de las Ciencias, v. 37, n. 1, p. 25-53, 2019.

VÁZQUEZ-BERNAL, B.; JIMENEZ PEREZ, R.; MELLADO, V.; TABOADA LEÑERO, M. C. The process of change in science teachers' professional development: a case study based on the types of problems in the classroom. Science Education, v. 96, n. 2, p. 337-363, 2012.

VERDUGO-PERONA, J.; SOLAZ-PORTOLÉS, J.; SANJOSÉ-LÓPEZ, V. El conocimiento didáctico del contenido en ciencias: estado de la cuestión. Cadernos de Pesquisa, São Paulo, v. 47, n. 164, p. 586-611, abr./jun. 2017.

NOTA: Todos los autores han contribuido igualmente en la elaboración y revisión de este artículo.

\section{CÓMO CITAR ESTE ARTÍCULO}

MELO, Lina; CABALLERO, Ana; SOTO-ARDILA, Luis Manuel; MELO, David. La dimensión curricular como componente del conocimiento didáctico del contenido. Cadernos de Pesquisa, São Paulo, v. 50, n. 175, p. 210-233, jan./mar. 2020. https://doi.org/10.1590/198053146526 


\begin{tabular}{|c|c|c|c|}
\hline & $\begin{array}{l}\text { TENDENCIA } \\
\text { TRADICIONAL }\end{array}$ & $\begin{array}{l}\text { TENDENCIA } \\
\text { INTERMEDIA }\end{array}$ & $\begin{array}{c}\text { TENDENCIA } \\
\text { CONSTRUCTIVISTA }\end{array}$ \\
\hline 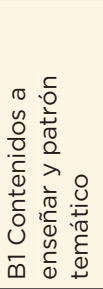 & $\begin{array}{l}\text { B1.1 } \\
\text { Los temas centrales que se } \\
\text { enseñan corresponden a } \\
\text { los propios de la física. Más } \\
\text { o menos interesantes para } \\
\text { el profesor. Son difíciles } \\
\text { de expresar de formas } \\
\text { más interesantes para los } \\
\text { estudiantes. }\end{array}$ & $\begin{array}{l}\text { B1.2 } \\
\text { Los contenidos como } \\
\text { transformación didáctica } \\
\text { de procesos y productos } \\
\text { disciplinares. }\end{array}$ & $\begin{array}{l}\text { B1.3 } \\
\text { Los contenidos como } \\
\text { integración y transformación } \\
\text { didáctica de lo cotidiano, } \\
\text { científico, histórico, social e } \\
\text { ideológico como contenido. Son } \\
\text { de interés para el profesor y } \\
\text { para los estudiantes. }\end{array}$ \\
\hline 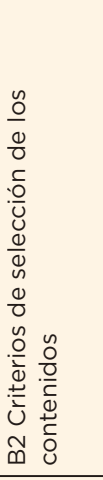 & $\begin{array}{l}\text { B2.1 } \\
\text { Lo que el currículo ha descrito } \\
\text { contribuye al acceso a la } \\
\text { universidad y en algunos casos } \\
\text { a la formación profesional } \\
\text { de los estudiantes; por tanto, } \\
\text { el profesor muestra gran } \\
\text { preocupación por alcanzar los } \\
\text { objetivos y la programación. } \\
\text { El tiempo y el examen de } \\
\text { selectividad es un criterio para } \\
\text { la selección de los contenidos } \\
\text { Van de lo simple a lo complejo. }\end{array}$ & $\begin{array}{l}\text { B2.2 } \\
\text { La selección de los contenidos } \\
\text { se da por experiencias exitosas } \\
\text { anteriores. } \\
\text { En los cursos de secundaria se } \\
\text { realiza un acercamiento a la } \\
\text { física a través de experimentos, } \\
\text { sin entrar en el formalismo } \\
\text { matemático del fenómeno. } \\
\text { Distinción entre el currículo de } \\
\text { secundaria y bachillerato. }\end{array}$ & $\begin{array}{l}\text { B2.3 } \\
\text { Tiene en cuenta la actitud } \\
\text { de las estudiantes frente } \\
\text { a la modificación de su } \\
\text { programación de contenidos. } \\
\text { Continuidad temática del } \\
\text { programa en función de los } \\
\text { objetivos de formación. } \\
\text { Procesos de reflexión del propio } \\
\text { profesor como criterio de } \\
\text { selección del diseño curricular. }\end{array}$ \\
\hline 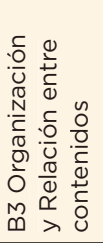 & $\begin{array}{l}\text { B3.1 } \\
\text { Versión actualizada y } \\
\text { simplificada del conocimiento } \\
\text { científico. }\end{array}$ & $\begin{array}{l}\text { B3.2 } \\
\text { Existe relación con otras } \\
\text { materias y contextos, pero se } \\
\text { mantiene una programación } \\
\text { rígida. }\end{array}$ & $\begin{array}{l}\text { B3.3 } \\
\text { Integración de lo académico con } \\
\text { lo contextual. } \\
\text { Centros de Interés } \\
\text { Perspectiva globalizadora }\end{array}$ \\
\hline 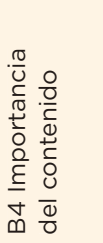 & $\begin{array}{l}\text { B4.1 } \\
\text { Preparar al estudiante para el } \\
\text { examen de selectividad y la } \\
\text { Universidad. }\end{array}$ & $\begin{array}{l}\text { B4.2 } \\
\text { En secundaria los contenidos } \\
\text { intentan dotar de sentido el } \\
\text { nuevo campo de conocimiento } \\
\text { que ve el estudiante. }\end{array}$ & $\begin{array}{l}\text { B4.3 } \\
\text { Mostrar el desarrollo y la } \\
\text { construcción de una teoría } \\
\text { física. }\end{array}$ \\
\hline 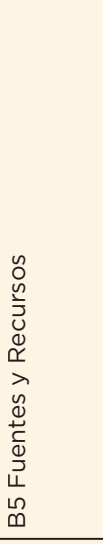 & $\begin{array}{l}\text { B5.1 } \\
\text { Las fuentes utilizadas } \\
\text { complementan la información } \\
\text { del libro de texto. Su selección } \\
\text { se ciñe a la lógica que indica } \\
\text { el plan de estudios y a una } \\
\text { exposición ordenada de los } \\
\text { contenidos. } \\
\text { Refuerzan la exposición del } \\
\text { profesor y hacen que la clase } \\
\text { se enriquezca y se vuelva } \\
\text { más interesante y atractiva. } \\
\text { En algunas ocasiones carecen } \\
\text { de criterios de selectividad } \\
\text { definidos. }\end{array}$ & $\begin{array}{l}\text { B5.2 } \\
\text { Se utilizan varias fuentes. Para } \\
\text { su selección se tienen en cuenta } \\
\text { las necesidades formativas } \\
\text { futuras del estudiante y los } \\
\text { resultados de las evaluaciones } \\
\text { externas/internas de los } \\
\text { estudiantes. } \\
\text { Recursos variados, amplían } \\
\text { el campo de la experiencia } \\
\text { del estudiante y facilitan la } \\
\text { aplicación, verificación y } \\
\text { elaboración de explicaciones. } \\
\text { Su implementación futura se } \\
\text { justifica en la lógica del ensayo } \\
\text { y error. }\end{array}$ & $\begin{array}{l}\text { B5.3 } \\
\text { Integración entre diversas } \\
\text { fuentes, algunas veces resultado } \\
\text { del consenso profesor- } \\
\text { estudiante. } \\
\text { Recursos flexibles y dinámicos, } \\
\text { se adaptan según el contexto } \\
\text { y permiten una amplia } \\
\text { comunicación entre profesor, } \\
\text { conocimiento y estudiantes. }\end{array}$ \\
\hline 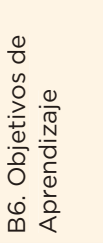 & $\begin{array}{l}\text { B6.1 } \\
\text { Conceptuales y procedimentales } \\
\text { cuyo fin es resolver y predecir } \\
\text { una situación planteada } \\
\text { utilizando algoritmos y } \\
\text { definiciones. }\end{array}$ & $\begin{array}{l}\text { B6.2 } \\
\text { Conceptuales y procedimentales } \\
\text { cuyo fin es potenciar las } \\
\text { observaciones cualitativas, y la } \\
\text { detección de regularidades. }\end{array}$ & $\begin{array}{l}\text { B6.3 } \\
\text { Los objetivos se extienden a } \\
\text { procedimientos y actitudes. } \\
\text { Son alcanzables y están en } \\
\text { consonancia con los contenidos, } \\
\text { las actividades y la evaluación } \\
\text { propuesta. }\end{array}$ \\
\hline
\end{tabular}

